\title{
Modified Anpr using Neural Networks
}

\author{
Shahala Shanavas, Sneha Raju, Sreeganesh S., Sreejil B.Nair, Albins Paul
}

\begin{abstract}
Number Plate Recognition is a mass observation technique which is used to identify the vehicles. The identification and acknowledgement of a vehicle license plate is a key method in the greater part of applications related to vehicle movement. Moreover, it is a very famous and dynamic research subject in the field of image processing. Since every vehicle have a unique plate number, so if we have to perceive a specific vehicle we can utilize the license plate. The main objective of automatic vehicle number plate recognition is to design an efficient automatic authorized vehicle identification system by using the number plate. It has three modules namely license plate extraction, segmentation and recognition. . Different methods, techniques and algorithms have been developed to detect and recognize license plates. Nevertheless, due to the license plate characteristics that vary from one country to another in terms of numbering system, colours, language of characters, fonts and size. Further investigations are still needed in this field in order to make the detection and recognition process very efficient. Although this domain has been covered by a lot of researchers, various existing systems operate under well-defined and controlled conditions. For example, some frameworks require complicated hardware to make good quality images or capture images from vehicles with very slow speed. For this reason the detection and recognition of number plates in different conditions and under several climatic variations remains always difficult to realize with good results. For that, we present an automatic system for number plate detection and recognition based on convolutional neural networks. CNN has proved its robustness even with distorted, tilted and illuminated datasets.
\end{abstract}

Keywords : ANPR, Image processing, number plate recognition, character segmentation, convolutional neural network, character recognition.

\section{INTRODUCTION}

Automatic vehicle number plate recognition is an automated system for recognizing license plate of vehicles. On Indian roads there are assortment of number plate formats and assortment of textual styles are utilized in vehicles and the most widely recognized vehicle number plate used is yellow or white as background and black used as foreground color. Each vehicle is equipped with an identity in the form of

Revised Manuscript Received on July 22, 2019.

Shahala Shanavas, ECE Dept., ASIET, Matoor, Kalady, Ernakulam683574, shahalashanavas916@gmail.com

Sneha Raju, ECE Dept., ASIET, Matoor, Kalady, Ernakulam683574,sneharaju971998@gmail.com

Sreeganesh S., ECE Dept., ASIET, Matoor, Kalady, Ernakulam683574, sreeganeshcitys@gmail.com

Sreejil B. Nair, ECE Dept., ASIET, Matoor, Kalady, Ernakulam683574, sreejilbnair@gmail.com

Albins Paul, Asst. Professor, ECE Dept., ASIET, Matoor, Kalady, Ernakulam-683574, , albins.ec@adishankara.ac.in a number plate. In light of the number plate code, the vehicle can be tracked ownership and rights to the vehicle.This is extremely helpful for the police in dealing with cases on the road. Inspection of vehicle identity by the police is usually done by checking the vehicle's documents. Document forgery is often found at the time of inspection. Along with the development of artificial intelligence technology an automated system for vehicle number plate recognition can be introduced. So automatic vehicle number plate recognition is an image processing technique which is used to perceive a vehicle without human intervention legitimately. Motivated by the feature learning capabilities of convolutional neural networks (CNN), the superior work is the identification and acknowledgment of the vehicle plate number image, which is accomplished by dint of Convolution Neural Network (CNN). Self-synthesized feature of CNN is capable of recognizing the states of the vehicle from the number plate with a sensibly high accuracy of $90 \%$ even with very low training size. CNN has proved its robustness even with distorted, tilted and illuminated datasets.

The proposed model can be partitioned into four parts 1) Grayscale conversion 2) Number plate extraction 3) Character segmentation 4) Character recognition. Here, the character recognition of vehicle number plates using Convolutional Neural Network (CNN) is one of the deep learning methods. The character recognition process is realized by the segmentation process, which is taking the characters in the number plate. The character recognition of vehicle number plates uses the method of deep learning. Researches that used CNN method have reached over $90 \%$ of accuracy.

ANPR systems have been implemented in many countries like Australia, Korea and few others. Strict implementation of license plate standards in these countries has helped the early development of ANPR systems. These systems use standard features of the license plates such as: dimensions of plate, border for the plate, color and font of characters, etc. that help to localize the number plate easily and identify the license number of the vehicle. In India, number plate standards are rarely followed. Wide variations are found in terms of font types, script, size, placement and color of the number plates. In few cases, other unwanted decorations are present on the number plate. Also, unlike other countries, no special features are available on Indian number plates to ease their recognition process. Hence, currently only manual recording systems are used and ANPR has not been commercially implemented in India.

\section{A. Challenges of ANPR in India:}

- Non Uniformity in License Plates depends on the Class of vehicles

- Single / Multi Line Characters in the License Plate 
- Dust / Unwanted Materials in the License Plate

- Non Uniformity in the Font Styles, Font Size and Color

- Plate inconsistency and jurisdictional differences.

- Accuracy and measurement of ANPR system performance.

- Speed of the vehicle.

- Incorrect cameras used.

- Weather conditions.

- Poor onsite traffic management resulting in missed plates/vehicles.

- Excessive skew angles causing recognition issues.

\section{RELATED WORKS}

During last couple of years different techniques and methods have been proposed for the efficient recognition of characters in the vehicle number plate. These includes the work already done by various researchers using different methodologies and algorithms. Some of the related works are as follows ;

OCR (Optical Character Recognition) scheme is applied for reading the image of vehicle number plate.In this system, a template matching technique is used in implementing the ANPR system for number plate recognition of vehicles. The objective of this system is to recognize the vehicle's number plate by matching the template scheme. In this system, firstly we capture the image of number plate then process it and read each and every character present in the number plate for their perfect recognition. The most significant phase is OCR, where the letterings on the image of number plate are changed into the texts which can be decoded later. The procedure of locating the region of a sub-photograph called a template, inside an picture. Matching of templates entails figuring out resemblances between a given template and home windows of the same size in an image and figuring out the window that produces the very best similarity degree. It works by comparing each and every pixel of the photograph and template for every feasible template displacement. This method involves the use and help of a database of characters or templates. For all feasible input characters there exists a template, for every alphanumeric characters templates are created (from A-Z and zero-nine) the use of 'regular' font style. The concept of ANPR system is based on the matching of templates and exactness of this system was established as $75-85 \%$ for Indian number plates[1].

In Sobel edge detection technique with morphological operation is used to detect the number plate characters, bounding box procedure is utilized to segment and extract each character from the number plate. When using Sobel Edge Detection, the image is processed in the $\mathrm{X}$ and $\mathrm{Y}$ directions separately first, and then combined together to form a new image which represents the sum of the $\mathrm{X}$ and $\mathrm{Y}$ edges of the image. When using a Sobel Edge Detector, it is first best to convert the image from an RGB scale to a Grayscale image. After the segmentation, template matching approach is made use of to match the numbers and characters of the number plate.Sobel Edge Detection is the fact that there tends to be a lot of noise in the final processed image[3].

Connected Components Analysis (CCA) are becoming increasingly popular in image processing techniques.
Character segmentation is the process of extracting the characters and numbers from the license plate image. Miscellaneous features make the character segmentation mission difficult, like image noise, plate frame, space mark, plates rotation and light variance. After removing the plate borders, then it starts with removing the noise from the plate. In binary image processing, CCA is an important technique that scans and labels the pixels of a binarized image into components based on pixel connectivity. Each pixel is labeled with a value depending on the component to which it was assigned. The connected components are then analyzed to filter out long and wide components and only left the components according to the defined values to extract the number plate. CCA convey imperative technique that scans and labels the pixels of a binarized image into components based on pixel connectivity Quantization, concerned in image processing, is a glossy compression technique achieved by compressing a range of values to a single quantum value. When the number of distinct symbols in a given stream is reduced, the brook becomes more compressible. As a result Vehicle plate segmentation from the vehicle engages in recreation is trouble-free and effectual task in recognizing the License plate. In prospect the researchers are so eminent to grip certain new attitude in recognizing the License plate[4].

Optical character recognition (OCR) to recognize the individual character with the help of database stored for each and every alphanumeric character. The camera is interfaced using Matlab with the PC. Different images of cars having different colors and structure types are taken and stored in PC. The images are in RGB format and the resolution is $800 \mathrm{x}$ 600 pixels. After capturing the image the next step was the yellow search algorithm. After the executing the yellow search algorithm the result contain the white region represents the yellow or color closer to the yellow. It can be observed that the yellow search algorithm successfully detect the ROI that only contain vehicle number plate. The smearing algorithm used next to extract the vehicle number plate. Once the vehicle number plate is extracted, it is converted into the binary format. The row and column segmentations methods are used next to extract the individual character in the vehicle number plate. Finally OCR is used for character recognition and each and every alphanumeric character is recognized. The OCR methods used in this project for the recognition is sensitive to misalignment and to different sizes, the affine transformation can be used to improve the OCR recognition from different size and angles. The statistical analysis can also be used to define the probability of detection and recognition of the vehicle number plate[2].

A number of studies have been conducted in various fields using deep learning. In particular, it is particularly noteworthy in the field of visual imaging, which is applied to objects detection, recognition, and tracking. The deep-learning algorithms, such as CNN (Convolutional Neural Network) have excellent performance compared to traditional algorithms in various fields. By leveraging the GPU, complex networks can also be deployed in real time. When a real-time image processing application is implemented with a general-purpose computer 
(CPU) is usually heavily loaded and in many cases that CPU alone cannot meet the real-time requirement at all. After conducting an in detail study, it was concluded that $\mathrm{CNN}$ is the best option for ANPR systems mainly in terms of efficiency and accuracy. Self synthesized feature of CNN is capable of recognizing the states of the vehicle from the number plate with a reasonably high accuracy of $90 \%$ even with very low training size. CNN has proved its robustness even with distorted, tilted and illuminated datasets. CNN can overcome most of the challenges faced by existing ANPR systems using template matching technique. Convolutional Neural Networks are composed of multiple processing layers to learn the representations of data with multiple abstract levels, are the most successful machine learning models in recent years. These models can have millions of parameters and many layers, which are difficult to train, and sometimes several days or weeks are required to tune the parameters. However, it can offer very high accuracy than any other existing methods for ANPR.

\section{OBJECTIVES}

The main objectives of the project are:

- To develop a better number plate recognition system than the conventional one.

- To reduce the vehicle crime rate and associated threats.

- To provide a better traffic monitoring system.

- To extend the accessibility of ANPR to various applications such as toll collection, parking, vehicle theft control and various other domains that are untouched by the conventional system.

\section{METHODOLOGY}

The proposed method consists of several steps to identify vehicle number plate.It have a camera that takes a picture, search the position of the number plate and then identifies the features of characters using character detection methods. Automatic number plate detection system is use in parking management and use for security purpose. The ANPR system has following steps :

- Vehicle image obtaining: Image of vehicle is taken.

- Pre-processing: Obtaining an estimated image and eliminate the distortions.

- Extraction of number plate: Remove irrelevant part and extract the number plate.

- Character segmentation: Segmentation or isolation of characters from the extracted license plate.

- Character recognition: Recognize letters and display the output result.

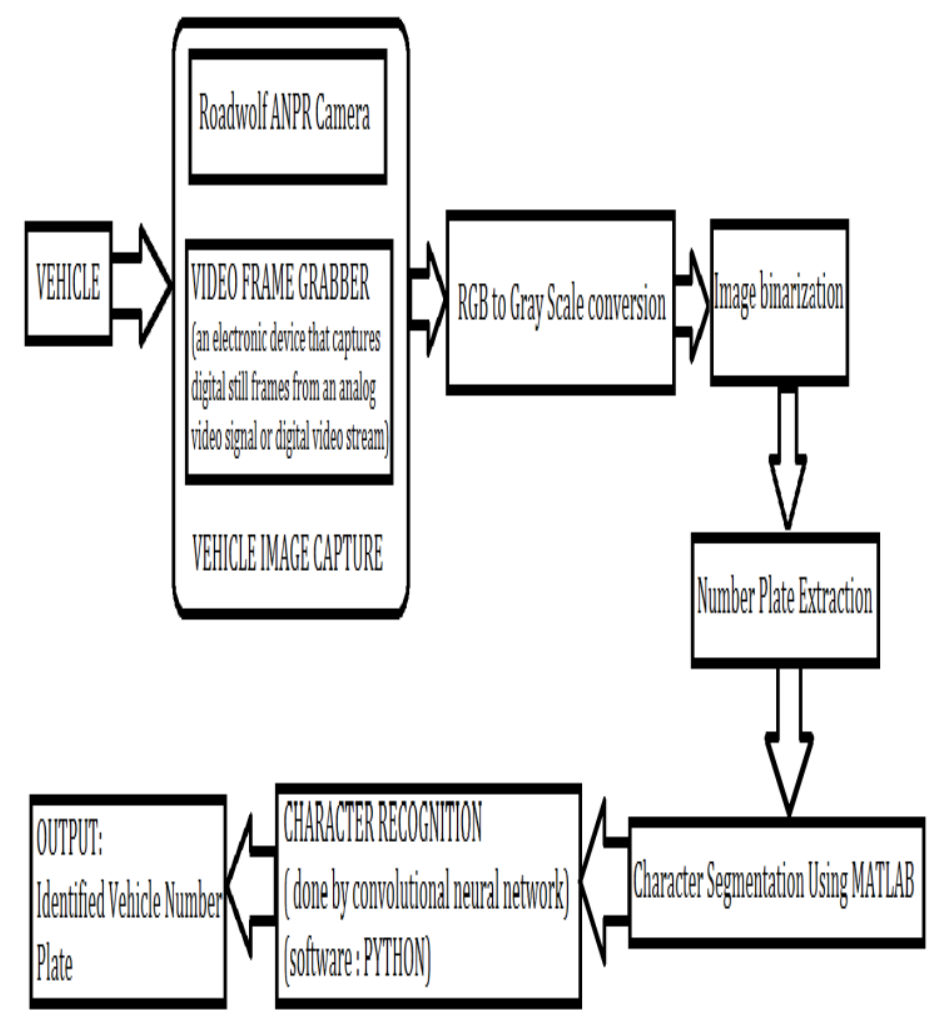

Figure 1: Block diagram of proposed system

\section{A. Vehicle Image Capturing}

For capturing vehicle number plate RoadWolf ANPR camera and video frame grabber are used.

- RoadWolf ANPR camera: The latest LED technology is used to deliver a new standard of number plate reading in RoadWolf cameras which is compact and light weighted range. The camera modules captures the corresponding images of vehicle in both daylight and challenging light conditions.

- Video frame grabber: Video frame grabber is an electronic device which captures digital still frames from an analog video signal or digital video stream. It can be stand-alone devices which plug into a port on the computer or a function built into the graphic cards. In video frame grabber video frames are captured in digital form and then displayed, stored, transmitted, analyzed, or combinations of these are take place.

\section{B. RGB to Gray scale conversion}

At first the image has been captured using a mobile camera or digital camera and then connected to the PC. The images are in RGB format and then it processed for the number plate extraction. Then we need to resize the image keeping the aspect ratio same.

Here, the RGB image is converted into Gray-scale image. The color image consists of three colors, $\operatorname{Red}(\mathrm{R}), \operatorname{Green}(\mathrm{G})$ and Blue(B) each consuming 8bits of information (i.e total 24-bit).We need to convert this 24-bit color image into 8-bit gray image using the formula written in equation(1). The Gray-scale transformation facilitates the plate extraction and increases the processing speed . $\mathrm{g}=0.299 \mathrm{R}+.587 \mathrm{G}+0.114 \mathrm{~B}$

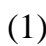




\section{Modified ANPR using Neural Networks}

\section{Image binarization}

We need to convert the gray scale image into binary (White and Black) image using a suitable threshold value for better output. So the resulting image consists only black and white pixels.to establish the uniformity in the dimensions of the input, the variable size image gets transformed into a binary matrix of fixed pre-determined dimensions, leading to reduce computational time .

\section{Number plate extraction}

After the detection of number plate area that area is extracted from the image. Firstly the row and column indices of plate area are found by connected component analysis. The extracted number plate may consist of various noise or unwanted holes. So enhancement of plate region is done. The result of plate region enhancement by morphological dilation, erosion, opening and closing operation on extracted plate region .

\section{E. Character segmentation}

After plate extraction, the next step is to isolate each characters from the image component. This step is called Character Segmentation. For this purpose Connected Component labelling (CCL) is used by which each characters are labelled uniquely and identify them in the next step. The CCA scans and labels the pixels of a binarized image component and every pixel is assigned with a value depending on the components. After that the connected components are analysed and remove the long and wide components and only left the components depends on the defined value. After this operation we get a set of foreground segments which are supposed to be characters and digits within the license plate.Character segmentation is done by using MATLAB.

\section{F. Character recognition}

After character segmentation, the next step is to recognize each character in the number plate. In the proposed system, the character recognition is done by Convolutional Neural Network.

A Convolutional Neural Network (CNN) formed by one or more convolutional layers and then followed by one or more fully connected layers. There are four main operation in convolutional Neural Network.They are Convolution, Non-linearity (RELUs), Pooling or Sub sampling and Classification (Fully Connected Layer). Character recognition process is done by PYTHON programming language.

\section{EXPERIMENTAL RESULTS}

Experiments have been performed to evaluate the efficiency of the proposed system.

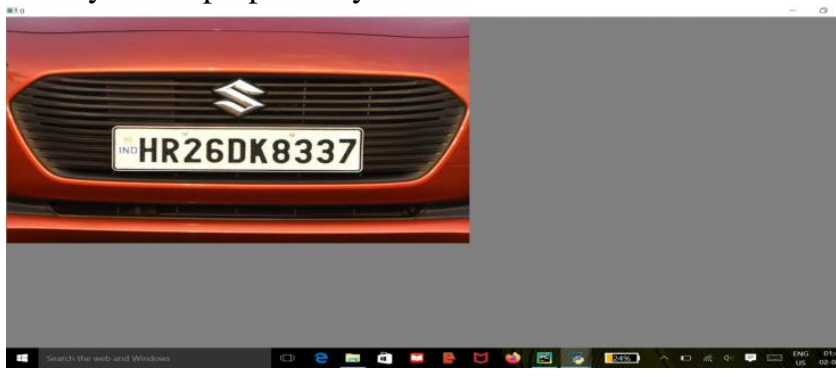

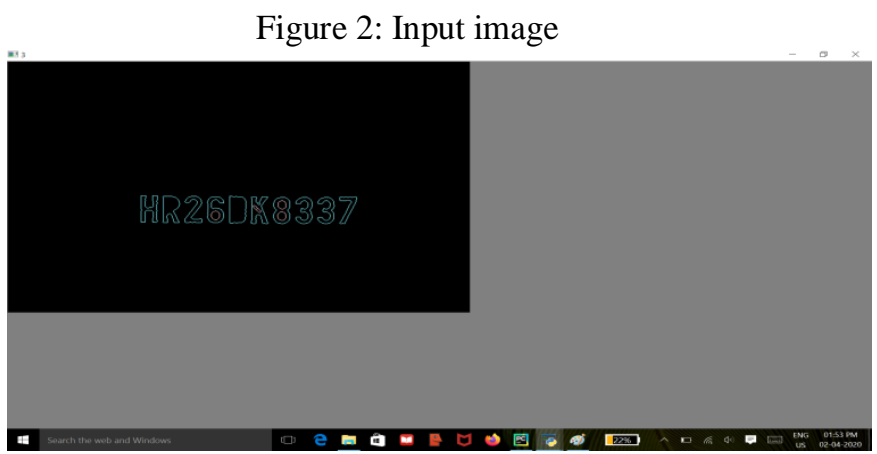

Figure 2: Final Output

Inorder to measure the accuracy of the system 25 color images were used as inputs, out of which the output of 21 images were found quite satisfactory. So the overall accuracy for the proposed approach is $84 \%$.

Table - I: Accuracy of proposed system

\begin{tabular}{|c|c|c|}
\hline APPROACH & $\begin{array}{c}\text { NUMBER OF } \\
\text { IMAGES }\end{array}$ & ACCURACY \\
\hline $\begin{array}{c}\text { Modified ANPR } \\
\text { using neural } \\
\text { networks }\end{array}$ & 25 & $84 \%$ \\
\hline
\end{tabular}

\section{CONCLUSION}

An efficient and convenient method for recognizing license plate based on Convolutional neural network have been proposed. The proposed system is useful in various scenarios such as parking system, vehicle theft control, vehicle speed monitoring, reducing traffic congestion, automatic toll tax collection etc. The system will capture the images of vehicles and extracts the number plate from the image automatically and then character segmentation and recognition will be performed inorder to recognize the characters written on the number plate.The $\mathrm{CNN}$ based number plate recognition system is found to be more efficient and accurate than all the other existing ANPR models.

Currently, ANPR works on Template Matching Technique which is prone to the following challenges: circumvention, plate inconsistency and jurisdictional differences, Accuracy and measurement of ANPR system performance, Speed of the vehicles, incorrect cameras, weather conditions, poor on site traffic management resulting in missed plates/vehicles, excessive skew angles causing recognition issues. These challenges faced by the existing system clearly demands for a better one. As a part of the literature survey, various IEEE publications were read and a study was conducted on convolutional neural network and found that $\mathrm{CNN}$ is the best technique in terms of accuracy and efficiency for an ANPR system.

Since the ANPR system using CNN includes various steps such as image acquisition, gray scale conversion, number plate extraction, character sementation and recognition, a study was conducted to 
identify what all are the requirements for each of the above mentioned steps. It was concluded that Roadwolf camera can be used to capture the image of number plate, along with that a video frame grabber can be used to convert analog video signal or digital video frame to digital still frames. Firstly, an Ultrasonic sensor is used to see if there's a car. If there is, it starts the camera. A SingleShotDetector mobilenetV2 deep learning object detection network is used to detect the position of number plate. The image is then preprocessed with openCV and uses CNN to extract text from image.If the number plate is verified, it will do face recognition. For that, it uses dlib library which is pretrained on 3 million images to generate encodings of face. Then, it is compared to the ones trained in the database. If verified, the garage door will open.Character segmentation can be done using MATLAB, by using the Regionprops function of MATLAB the characters of the resulted number plate region are separated which gives us the defined boxes for each of the characters. The smallest defined box that contains a character is returned by Regionprops function. This method is used to obtain the defined boxes of all characters in the number plate. The character recognition can be done using PYTHON.CNN is used to recognize each character in the number plate and to classify them to various classes in the dataset. Future Scopes of the project are;

- Improving motion detection: set a slight delay in the image capture. So rather than immediately capturing the image, wait 0.5 seconds for the vehicle to be more prominent. This way, the number plate can be more prominent in the photo for the ANPR engine.

- Improving camera focus : adjust the camera position and width of focus so that the license plate can be more prominent.

\section{REFERENCES}

[1] Surajit Das, Joydeep Mukherjee, " Automatic License Plate Recognition Technique using Convolutional Neural Network", International Journal of Computer Applications Volume 169 - No.4, July 2017.

[2] Muhammad TahirQadri,MuhammadAsif," Automatic number plate recognition system for vehicle identification using optical character recognition", International Conference on Advances in Computing, Communication Control and Networking (ICACCCN2018)

[3] Faizalpatel,JaiminiSolanki,VivekRajguru,AnkitSaxena"Recognition of Vechicle Number Plate Using Image processing Technique" Advanced Emergency Medicine 2018

[4] S. Stephy Golda Mercy, Dr. I. MuthulakshmiPG Scholar,"Automatic number plate recognition using connected component analysis algorithm", International Journal For Technological Research In Engineering Volume 5, Issue 7, March-2018

[5] Jiudong Yang, Jianping Li, "Application of deep convolution neural network ", International Centre for Wavelet Analysis and Its Applications.

[6] PrathameshKulkarni,AshishKhatri, PrateekBanga, Kushal Shah," Automatic Number Plate Recognition (ANPR) System for Indian conditions"

[7] TianmeiGuo, Jiwen Dong ,HenjianLi'YunxingGao , “Simple Convolutional Neural Network on Image Classification", Department of Computer Science and Technology Shandong Provincial Key Laboratory of Network based Intelligent Computing University of Jinan, Jinan, China,2017

[8] SaadAlbawi ,Tareq Abed Mohammed, Saad Al-Zawi, "Understanding of a Convolutional Neural Network", Department of Computer Engineering, Istanbul University, Turkey, Department of Electronic Engineering, Diyala University, 2017.

\section{AUTHORS PROFILE}

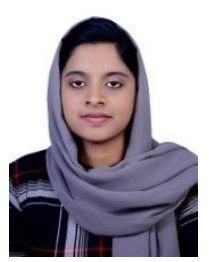

Shahala Shanavas, Department of Electronics and Communication Engineering ,Adishankara Institute of Enginering and Technology, Matoor, Kalady, Ernakulam - 683574. shahalashanavas916@gmail.com

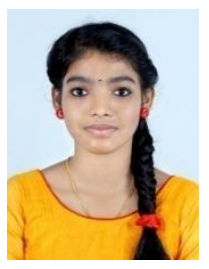

Sneha Raju, Department of Electronics and Communication Engineering ,Adishankara Institute of Enginering and Technology, Matoor, Kalady, Ernakulam - 683574 sneharaju971998@gmail.com

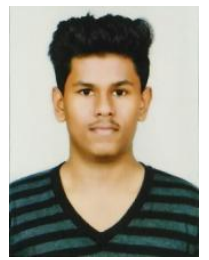

Sreeganesh S., Department of Electronics and Communication Engineering ,Adishankara Institute of Enginering and Technology, Matoor, Kalady, Ernakulam - 683574.

sreeganeshcitys@gmail.com

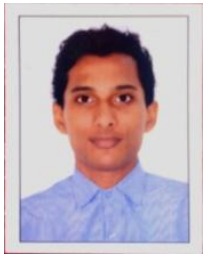

Sreejil B.Nair, Department of Electronics and Communication Engineering ,Adishankara Institute of Enginering and Technology, Matoor, Kalady, Ernakulam - 683574

sreejilbnair@gmail.com 